\title{
Fast Self-Tuning Decentralized Variable Frequency Optimal Controller Design for Three-Phase Embedded Grids
}

\author{
David Dewar $^{\dagger}$, Jaime Rhoten*, Andrea Formentini ${ }^{\dagger}$, Pericle Zanchetta ${ }^{\dagger}$ \\ ${ }^{\dagger}$ Department of Electrical and Electronic Engineering, The University of Nottingham, United Kingdom \\ ${ }^{*}$ Department of Electrical and Electronic Engineering, Universidad del Bío-Bío, Chile \\ David.Dewar@nottingham.ac.uk
}

\begin{abstract}
In this paper, a novel approach to variable frequency optimal controller design for the application of aircraft embedded grids is presented. Aircraft power grids by industrial standards need to be able to operate across a range of frequencies. Often, these systems are controlled by active power-based controls, which are well known for being rather slow in response time. A novel method of variable frequency optimal control using $\mathrm{H}_{2}$ optimization is presented. It is shown that designing the control as such not only guarantees the optimal performance across the full range of frequencies being designed for, but fast responses to frequency and step load changes can be achieved.

Index Terms-Optimal Control, Embedded Grids, Variable Frequency, Self Tuning, Three-Phase
\end{abstract}

\section{INTRODUCTION}

In areas such as More Electric Aircraft (MEA), three-phase embedded electrical systems are becoming more prevalent [1]. These systems comprise of multiple power converter systems interfaced with one another through a series of passive filter circuits. Electrical systems on aircraft must adhere to regulations set out in MIL-STD-704, which for variable frequency systems states the electrical system must adhere to a frequency range of $360-800 \mathrm{~Hz}$. Many studies that investigate the optimal control of such systems primarily investigate with respect to a nominal frequency of operation, in general, $400 \mathrm{~Hz}$ [2], [3]. However, these approaches cannot guarantee optimal performance and stability when operating at other frequencies in the required range. Another feature that is trying to be addressed across MEA is to develop smaller, lighter and more efficient electrical systems for aircraft. With multiple converters interconnected onto the same bus, converter cross-interactions become prevalent. The common method for mitigating these cross-interactions is to increase the passive filters between subsystems. This is counter-intuitive however, as this results in the increased sizing and weight to the system. Work into $H_{2}$ optimization to synthesize decentralized global optimal controls have shown to be able to mitigate the problem of subsystem interactions under reduced passive filters [4]. This paper proposes a method for which $\mathrm{H}_{2}$ optimization as shown in [2], [4] can be adapted such that optimal and stable performance can be guaranteed globally across the required frequency range. Other methods of optimal, nonlinear controllers exist which to some degree could handle the proposed problem. Examples being Model Predictive Control

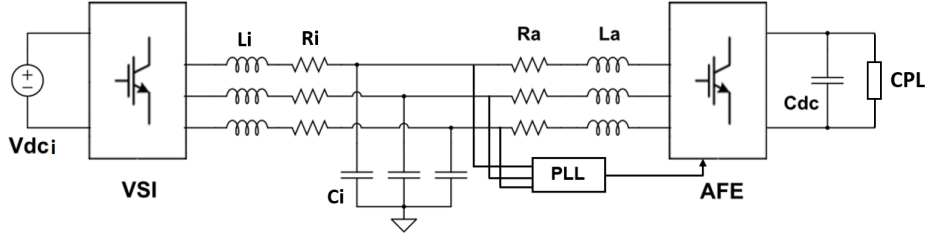

Fig. 1: The Notional Test System

(MPC) and Sliding mode controls (SMC). [5], [6] MPC, however, is very computationally heavy, resulting in increased costs for hardware implementation. SMC on the other hand, sacrifices performance, for a sub-optimal solution to incorporate decentralization of the controllers. [5] Decentralization being vital to reduce required cross-communication between sub-systems and to increase system reliability. Therefore, $\mathrm{H}_{2}$ control has been selected for use in this study. The proposed $\mathrm{H}_{2}$ controller not only guarantees stability and good performance against parametric uncertainty intrinsically, but will also be shown to be non-computationally heavy, but can also deliver fast dynamic performance to large step disturbances. Additionally, optimal performance can be guaranteed even when decentralizing the controller, which in turn results in the additional benefit of mitigation of cross-converter interactions.

\section{Notional System Model}

The notional system which is to be used in this study can be observed in Figure 1. The notional system includes a threephase Voltage Source Inverter (VSI) fed by a DC source and an LCR filter on its output. The Active Front End (AFE) is fed by the VSI generated grid through an RL filter. The AFE output has a DC-Link capacitor and a Constant Power Load (CPL). A CPL is used to exaggerate the non-linearities of the system, as well as to emulate industrial loads.

\section{A. State-Space Models of Notional System}

A common approach to designing controls for AC systems is to convert the system it the DQ frame, whereby an AC system can be modeled as two coupled DC systems, where simpler DC controls can then be implemented for the AC system. By following the method shown in [7] can the statespace equations of the system be constructed such that: 


$$
\dot{x}=A x+B u=\left[\begin{array}{cc}
A_{v s i} & \eta_{v} \\
\eta_{a} & A_{a f e}
\end{array}\right] x+\left[\begin{array}{cc}
B_{v s i} & 0 \\
0 & B_{a f e}
\end{array}\right] u
$$

Where, $A$ and $B$ define the state matrix and the input matrix for each subscripted converter respectively, and $\eta$ signifies cross-coupling terms between converters. The other terms are:

$$
\begin{aligned}
x= & {\left[\begin{array}{lll}
x_{v s i} & \mid & x_{a f e}
\end{array}\right]^{T} } \\
& =\left[\begin{array}{lllllll}
i_{i d} & V_{c d} & i_{i q} & V_{c q} & i_{a d} & i_{a q} & V_{d c_{a}}
\end{array}\right]^{T} \\
u & =\left[\begin{array}{llllll}
u_{v s i} & u_{a f e}
\end{array}\right]^{T}=\left[\begin{array}{lllll}
m_{d} & m_{q} & p_{d} & p_{q}
\end{array}\right]^{T}
\end{aligned}
$$

The subscripted $d q$ terms referring to either $d$ or $q$ axis. $i_{i d / q}$ refers to the VSI currents across the output filter inductor, and $V_{c d / q}$ refers to the VSI voltages across the output capacitor. $i_{a d / q}$ are the currents across the input filter inductor of the AFE, and $V_{d c_{a}}$ is the DC-Link voltage. The input terms $m_{d / q}$ and $p_{d / q}$ refer to the dq axis modulation index of the VSI and AFE respectively. The following terms which are to be used in this paper are as follows. $R_{i}, L_{i}$, and $C_{i}$ refer to the VSI filter resistance, inductance and capacitance respectively. The terms $R_{a}, L_{a}$ and $C_{a}$ refer to the AFE input filter resistance and inductance, and the DC-Link capacitor respectively. $P_{l}$ shall be used for the desired output load power on the AFE side.

\section{B. PLL Modelling and Controller Implementation}

When modeling and controlling systems in the DQ-Frame, the Synchronous Reference Frame Phase-Locked Loop (SRFPLL) is a commonly employed system which is fundamental to ensure good power delivery and synchronization across the multi-converter system. From Fig. 1 it can be seen augmented into the AFE control architecture. This form of PLL is very well studied, and thus will not be discussed in this paper, and the reader is referred to [2] for further information. The PLL has been designed to a bandwidth of $500 \mathrm{~Hz}\left(\omega_{P L L}\right)$, and to a damping ratio $(\zeta)$ of 0.7 , according to the pole placement equations below [2]:

$$
K_{p}=\frac{2 \zeta \omega_{p l l}}{V_{c d}} \quad K_{i}=\frac{\omega_{p l l}^{2}}{V_{c d}}
$$

\section{Controller Design And ConcePt}

Controller design techniques for which $H_{2}$ based methods have been used to synthesize optimal controls are not new, and with the advancement in research into optimal controls, they are being more widely than ever since its conception [8]. Within the field of optimal control of three-phase converters, most applications where $\mathrm{H}_{2}$ optimization, or related techniques, have been performed primarily focusing on single converter applications [9], [10], and also a few studies have been conducted looking into larger micro-grid applications [2] [7]. However, a key aspect of this optimization technique that has not yet been explored is to use this to develop an optimal variable frequency control which ensures optimal and stable performance across a to-be-designed for frequency range. This section highlights the design strategy used to develop this controller.

\section{A. Standard Decentralized $\mathrm{H}_{2}$ Controller}

The $\mathrm{H}_{2}$ controller synthesis permits the development of decentralized controls for the system (1). Decentralization of the control is important to reduce the required communication between the converters, along with building in redundancy to the system by ensuring subsystem controls depend only on states for the given converter subsystem. It is also desired for the system to run with zero steady-state error, and therefore extension to the system states $x$ with integral states is required, for each controlled state. This extension results in the following system described in (5), where $\omega$ states are newly included integrals to their subscripted states.

$$
\begin{aligned}
& \tilde{x}=\left[\begin{array}{llllll}
x_{v s i} & \omega_{V_{c d}} & \omega_{V_{c q}} & x_{a f e} & \omega_{i_{i q}} & \omega_{V_{d c_{a}}}
\end{array}\right]^{T} \\
& P=\left\{\begin{array}{c}
\tilde{x}=\tilde{A} \tilde{x}+B_{1} w+B_{2} u \\
z=\left[\begin{array}{c}
\sqrt{Q_{\gamma}} \\
0
\end{array}\right] \tilde{x}+\left[\begin{array}{c}
0 \\
\sqrt{R_{\gamma}}
\end{array}\right] u
\end{array}\right.
\end{aligned}
$$

The equations used to encapsulate the $H_{2}$ problem for the controller synthesis is shown in (6) where $\tilde{A}$ is the original A matrix extended with integral states. The signals $v, w$ and $z$ are the controller outputs, plant disturbances, and plant performance output respectively. The matrix $B_{1}$ is the disturbance matrix, detailing each of the states' disturbance influence to the system plant. As all states can be disturbed, this is set to an identity matrix. $B_{2}$ is the input matrix. $Q_{\gamma}$ and $R_{\gamma}$ are the controller weightings for the states and inputs respectively derived from the Riccati equation [2]. $Q_{\gamma}$ is selected to only weight the integral states of the system in order to dictate the speed to steady-state error after system disturbance. The larger the weight given, the faster the bandwidth to the associated state. $R_{\gamma}$ weights each of the inputs, as described in (3), and dictates the size of the control effort permitted to each input to correct error from disturbance. The larger the associated weight to an input, the less control effort given, and thus decreases overall controller bandwidth. The subsequent control law being composed as shown in (7) shows the imposed structural constraint on the controller optimization.

$$
u=K \tilde{x}=\left[\begin{array}{cc}
K_{v s i} & 0 \\
0 & K_{a f e}
\end{array}\right] \tilde{x}
$$

Where $K_{v s i} \in \Re^{2 \times 6}$ and $K_{a f e} \in \Re^{2 \times 5}$ are the to be synthesized controller gains for each converter. The process in order to synthesize the gains $K$ is to solve the structured $H_{2}$ optimization problem, for which the transfer function between the disturbances $w$ and the performance output $z$ is minimized by solving the following cost function (8).

$$
\begin{aligned}
& K=\min _{K} \\
& \sqrt{\int_{0}^{\infty} \operatorname{tr}\left(B_{1}^{\prime} e^{\left(\tilde{A}-B_{2} K\right)^{\prime} t}\left(Q+K^{\prime} R K\right) e^{\left(\tilde{A}-B_{2} K\right) t} B_{1}\right) d t}
\end{aligned}
$$

The gain structure in (7) enforces linear constraints on $K$ such that the optimization problem in (8) becomes of the form:

$$
K \in S
$$


Where $S$ is a subspace containing all the possible gains of $K$ which stabilizes the closed-loop system. The integral in (8) can also be shown to be in equivalent form to the following Lyapunov equation:

$$
\left(A-B_{2} K\right)^{\prime} P+P\left(A-B_{2} K\right)=-\left(Q+K^{\prime} R K\right)
$$

which in turn implies the following:

$$
J(K)=\operatorname{tr}\left(B_{1}^{\prime} P B_{1}\right)
$$

This cost function (11) develops a smooth function of $K$, as a result of the exponential and polynomial terms of $K$ in (8). Each $K$ ensures the system is asymptomatically stable for the given closed-loop system. Therefore it becomes possible to employ a gradient-based approach to solve the optimization in (8) such that:

$$
\nabla J(K)=\left(B_{2}^{\prime} P+R K\right) \Psi
$$

where $\Psi$ is the solution to the Lyapunov equation such that:

$$
\left(A-B_{2} K\right) \Psi+\Psi\left(A-B_{2} K\right)=B_{1} B_{1}^{\prime}
$$

Due to the non-convex nature of the optimization, a multiple starting point approach must also be employed to avoid local minima.

\section{B. $\mathrm{H}_{2}$ Variable Frequency Controller}

This section details the methodology to adapt the above method into a variable frequency control. The controller is designed to a specific frequency by selection of $\omega_{n}$ in the state equations. As a brief example the state equation for $i_{i d}$ for the VSI is shown in (14).

$$
\dot{i_{i d}}=-\frac{R}{L} i_{i d}-\frac{1}{L}+\omega_{n} i_{i q}+\frac{m_{d}}{2 L} V_{d c_{i}}
$$

Due to space limitations the all the state equations are not presented in this work but are all fully described in [2]. The state-space equations from which can be derived to be:

$$
\begin{gathered}
\tilde{A}=\left[\begin{array}{cc}
\tilde{A_{v s i}} & \eta_{v} \\
\eta_{a} & \tilde{A_{a f e}}
\end{array}\right] \quad B_{2}=\left[\begin{array}{ccccc}
B_{2_{V S I}} & 0 & \\
0 & B_{2_{A F E}}
\end{array}\right] \\
\tilde{A_{v s i}}=\left[\begin{array}{cccccc}
-\frac{R_{i}}{L_{i}} & -\frac{1}{L_{i}} & \omega_{n} & 0 & 0 & 0 \\
\frac{1}{C_{i}} & 0 & 0 & \omega_{n} & 0 & 0 \\
-\omega_{n} & 0 & -\frac{R_{i}}{L_{i}} & -\frac{1}{L_{i}} & 0 & 0 \\
0 & -\omega_{n} & \frac{1}{C_{i}} & 0 & 0 & 0 \\
0 & -1 & 0 & 0 & 0 & 0 \\
0 & 0 & 0 & -1 & 0 & 0
\end{array}\right]
\end{gathered}
$$

$$
\begin{gathered}
\tilde{A_{a f e}}=\left[\begin{array}{ccccc}
-\frac{R_{a}}{L_{a}} & \omega & -\frac{p_{d}}{2 L_{a}} & 0 & 0 \\
-\omega & -\frac{R_{a}}{L_{a}} & -\frac{p_{q}}{2 L_{a}} & 0 & 0 \\
\frac{3 p_{d}}{4 C_{a}} & \frac{3 p_{q}}{4 C_{a}} & \frac{P_{L}}{C_{a} V_{d c_{a}}^{2}} & & \\
0 & -1 & 0 & 0 & 0 \\
0 & 0 & -1 & 0 & 0
\end{array}\right] \quad(17) \\
\eta_{v}=\left[\begin{array}{ccccc}
0 & 0 & 0 & 0 & 0 \\
-\frac{1}{C_{a}} & 0 & 0 & 0 & 0 \\
0 & 0 & 0 & 0 & 0 \\
-\frac{1}{C_{a}} & 0 & 0 & 0 & 0 \\
0 & 0 & 0 & 0 & 0 \\
0 & 0 & 0 & 0 & 0
\end{array}\right] \quad \eta_{a}=\left[\begin{array}{cccccc}
0 & \frac{1}{L_{a}} & 0 & 0 & 0 & 0 \\
0 & 0 & 0 & \frac{1}{L_{a}} & 0 & 0 \\
0 & 0 & 0 & 0 & 0 & 0 \\
0 & 0 & 0 & 0 & 0 & 0 \\
0 & 0 & 0 & 0 & 0 & 0
\end{array}\right] \\
B_{2_{V S I}}=\left[\begin{array}{cc}
\frac{V_{d} c}{2 L} & 0 \\
0 & 0 \\
0 & \frac{V_{d} c}{2 L} \\
0 & 0 \\
0 & 0 \\
0 & 0
\end{array}\right] B_{2_{A F E}}=\left[\begin{array}{cccc}
-\frac{V_{d c_{a}}}{2 L_{a}} & 0 \\
0 & -\frac{V_{d c a}}{2 L_{a}} \\
\frac{3 I_{a d}}{4 C_{a}} & \frac{3 I_{a q}}{4 C_{a}} \\
0 & 0 \\
0 & 0
\end{array}\right] \quad(19)
\end{gathered}
$$

By selection of $\omega_{n}$, an optimal controller is then developed for that given grid frequency. Therefore, to incorporate the $\mathrm{H}_{2}$ optimization to a variable frequency controller synthesis, several controllers at multiple frequencies can be computed and interpolated. The performance of the global controller is dictated by the weighting matrices $Q_{\gamma}$ and $R_{\gamma}$. The initial step to this controller design is setting $R_{\gamma}$ to an identity matrix, and applying a weight of ' 1 ' to each element associated with an integral state in $Q_{\gamma}$. By analyzing the performance of this system with these weights, the $R_{\gamma}$ matrix is then tuned such that the converters are in the region of desired operable performance. In general, the larger the weight in $R_{\gamma}$, the slower the associated input, and vice versa. Thus, increasing $R_{\gamma}$ decreases closed-loop bandwidth. Then tuning $Q_{\gamma}$ can be used to fine-tune the performance state-by-state. Where increasing the gain to associated integral states increases its dynamic speed. Thus, increasing $Q_{\gamma}$ increases overall controller bandwidth. If a desired response of the control can be attained for both $\omega_{n_{\min }}$ and $\omega_{n_{\max }}$ independently, it can be ensured that an interpolated control will operate with desired performance across all frequencies. As an example below, Fig. 2 shows the performance of the system at $300 \mathrm{~Hz}$ and $800 \mathrm{~Hz}$, using the weights and system parameters specified in Table I. Only AFE performance shown due to space limitations.

In Fig. 2 a $1 \mathrm{~kW}$ step load is applied from no load to analyze the non-linear system dynamics. At both frequencies, it is clear a stable, and fast dynamic response can be achieved, which concludes that using the $Q_{\gamma}$ and $R_{\gamma}$ weights from Table I should result in a stable control with the same relative dynamics observed across the full frequency range. From the simulations, it can be observed that the grid voltages are largely unaffected by the disturbances. The grid being dictated by the VSI shows the subsystem cross-coupling mitigation achieved by this approach of controller design due to designing the control with full knowledge of the system dynamics described from $\tilde{A}$ in (8). 
TABLE I: System Parameters

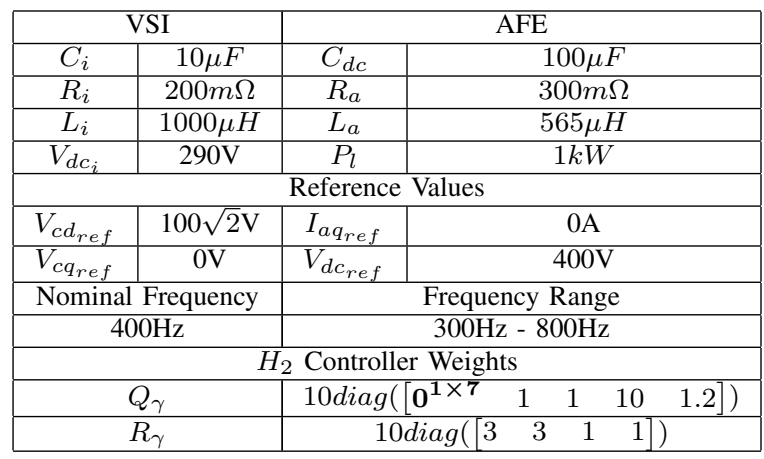
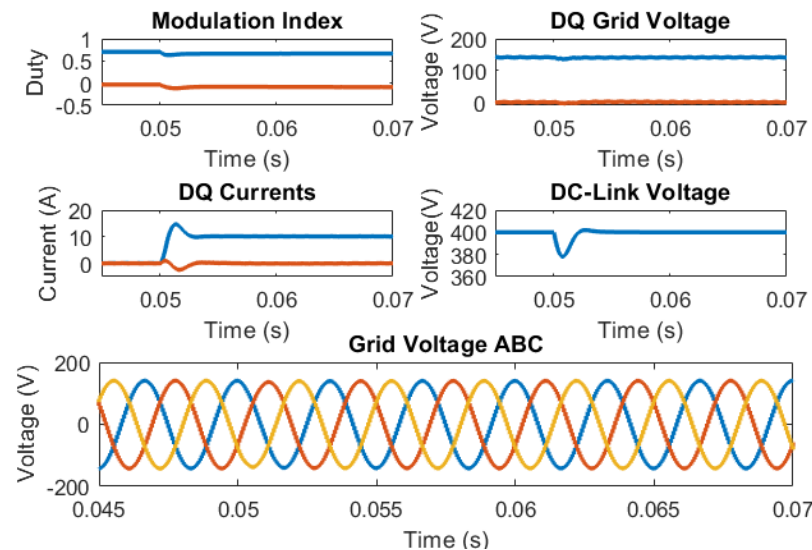

(a) Performance at $300 \mathrm{~Hz}$
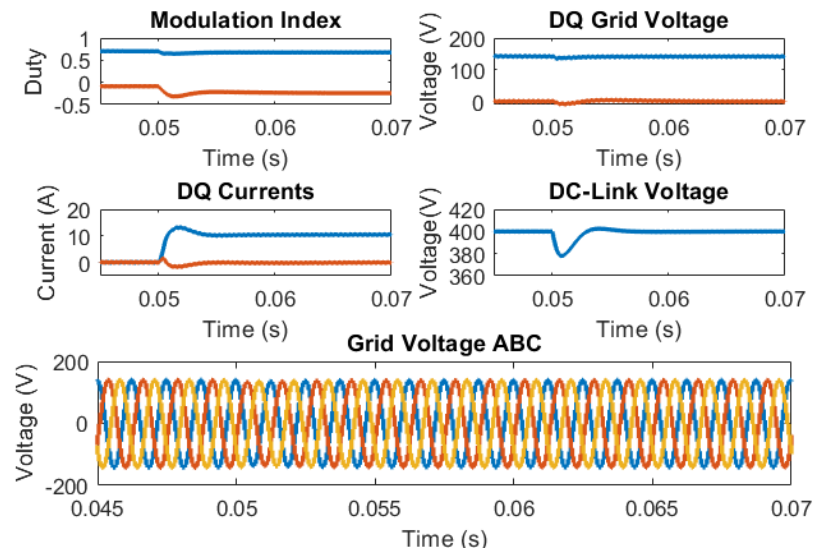

(b) Performance at $800 \mathrm{~Hz}$

Fig. 2: Min (a) and Max (b) frequency performance for controller validation

The intention of this controller design is to interpolate several controllers to derive an expression for the relation between frequency and the controller gains in $K$. Therefore, a gain resolution for the control needs to be selected. The greater the resolution of the interpolation, the better the performance, and optimality of the control across the frequency range. The drawback being increased computation time for the controller synthesis at higher resolutions. A resolution of $0.5 \mathrm{~Hz}$ was selected for this study. Let this term for the resolution value be $N_{\text {res }}=1000$. Therefore, $N_{\text {res }}+1$ controllers are going to be synthesized using the controller synthesis described. Starting
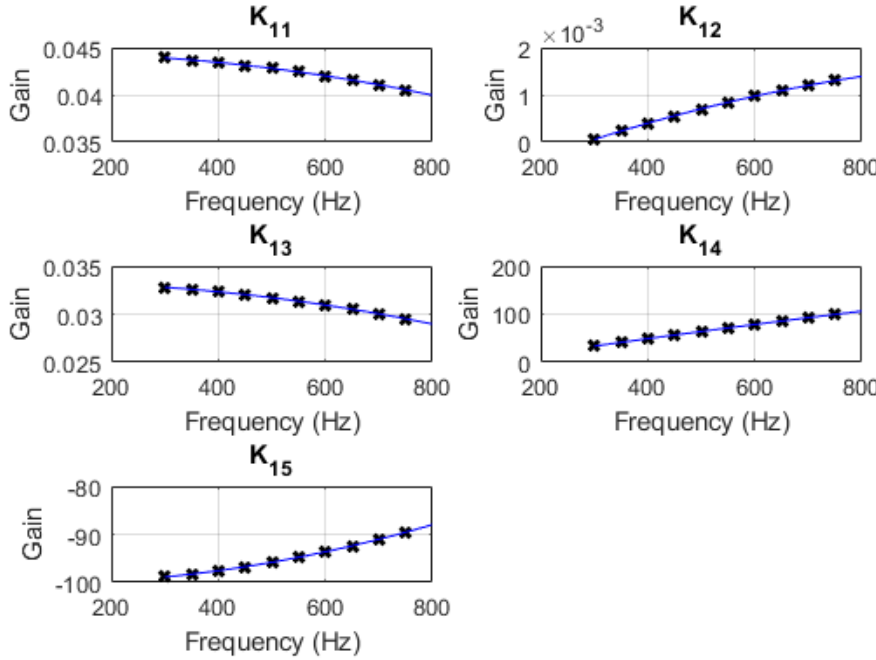

Fig. 3: Variations of first row gains of $K_{a f e}$ with respect to frequency, with interpolated gain function behaviour

at $\omega_{n_{\min }}$ the controller is first synthesized using $Q_{\gamma}$ and $R_{\gamma}$. This controller is saved into an array, and the synthesis loop increases the frequency by $0.5 \mathrm{~Hz}$ and develops the new control for this frequency and repeats until $\omega_{n_{\max }}$ has been reached.

The algorithm, therefore, results in $N_{\text {res }}$ controllers. One can then realize from Fig. 3 how the gains of $K$ change as frequency increases.

$$
K_{a f e}=\left[\begin{array}{lllll}
K_{11} & K_{12} & K_{13} & K_{14} & K_{15} \\
K_{21} & K_{22} & K_{23} & K_{24} & K_{25}
\end{array}\right]
$$

As can be observed, some gains vary greatly with the increase in frequency, and others remain relatively static. It is also very clear across all gains, each function can easily be interpolated into a $2^{\text {nd }}$ order polynomial with respect to frequency.

\section{Interpolation Method Description}

The interpolation method to get the controller gains (20) as a function of the actual frequency is based on interpolating a second order polynomial of the form $\widehat{K}_{i j}=a_{0}^{i j}+a_{1}^{i j}$. $f+a_{2}^{i j} \cdot f^{2}$, with $i=\{1,2\}$ and $j=\{1, . ., 6\}$ for VSI, and $j=\{1, . ., 5\}$ for AFE accordingly, where the parameters $a_{n}^{i j}$, for $n=\{0,1,2\}$, are obtained by using the Least Square Optimization Method, and $\widehat{K}_{i j}$ is the interpolated function of $K_{i j}$. For every $K_{i j}(f)$ a set of points is computed using the Hifoo toolbox [11] as a function of the frequency and then, the second order polynomial is adjusted with the optimal constants $a_{n}^{i j}$ and the real values $K_{i j}$ as shown in (21):

$$
K_{i j}(f)=\left[\begin{array}{lll}
1 & f & f^{2}
\end{array}\right]\left[\begin{array}{lll}
a_{0}^{i j} & a_{1}^{i j} & a_{2}^{i j}
\end{array}\right]^{T}
$$

Equation (21) is calculated for the specified frequencies between $f_{1}=300 \mathrm{~Hz}$ and $f_{N_{\text {res }}}=800 \mathrm{~Hz}$, and the optimal 
constants $a_{n}^{i j}$ are found as:

$$
\begin{aligned}
& \left.\left[\begin{array}{l}
a_{0}^{i j} \\
a_{1}^{i j} \\
a_{2}^{i j}
\end{array}\right]=\left[\begin{array}{ccc}
1 & f_{1} & f_{1}^{2} \\
1 & f_{2} & f_{2}^{2} \\
\vdots & \vdots & \vdots \\
1 & f_{N_{\text {res }}} & f_{N_{\text {res }}}^{2}
\end{array}\right]^{T}\left[\begin{array}{ccc}
1 & f_{1} & f_{1}^{2} \\
1 & f_{2} & f_{2}^{2} \\
\vdots & \vdots & \vdots \\
1 & f_{N_{\text {res }}} & f_{N_{\text {res }}}^{2}
\end{array}\right]\right]^{-1} \\
& {\left[\begin{array}{ccc}
1 & f_{1} & f_{1}^{2} \\
1 & f_{2} & f_{2}^{2} \\
\vdots & \vdots & \vdots \\
1 & f_{N_{\text {res }}} & f_{N_{\text {res }}}^{2}
\end{array}\right]^{T}\left[\begin{array}{c}
K_{i j}\left(f_{1}\right) \\
K_{i j}\left(f_{2}\right) \\
\vdots \\
K_{i j}\left(f_{N_{\text {res }}}\right)
\end{array}\right]}
\end{aligned}
$$

This results in a decentralized $2^{\text {nd }}$ order polynomial feedback gain matrices being produced for each converter. The first advantage of this approach is the self-tuning nature of the controller. As the frequency of the grid changes, the gains adjust accordingly to ensure optimal performance across all frequencies. This leads to the second advantage being that huge numbers of controllers need not be implemented on hardware, as is usual with similarly related gain scheduling techniques. Polynomial functions can instead be used to describe the change of gains with respect to frequency as an offline, self-tuning optimal control strategy. This, therefore means cheaper and less computationally heavy solutions to controller implementation can be employed. The interpolated polynomial gains are shown in blue in Figure 3.

\section{Simulation Results and Performance COMPARISON}

The proposed controller shall be compared to a more traditional PI control, to assess performance. The transfer functions and design method for the PI controller are presented in [4]. The PI transfer functions are not frequency dependent and thus do not need adaptation and can work across the full range of frequencies. Cascaded control loops are used to incorporate the current and voltage controls for each converter, as presented in [4]. By convention, inner current loops have 10x the bandwidth of the outer voltage loops and are designed as such. However, for overall best performance, it was found a ratio between bandwidths for AFE controller had to be 20x, and this was used henceforth. The bandwidths used to design the PI controllers in the study are shown in Table II. The bandwidths selected were the fastest possible with stable noncirculatory performance, at damping of 0.7 .

TABLE II: PI Controller Bandwidths for Comparisons

\begin{tabular}{|c|c|c|}
\hline & VSI & AFE \\
\hline$f_{I}$ & $1 \mathrm{kHz}$ & $800 \mathrm{~Hz}$ \\
\hline$f_{V}$ & $100 \mathrm{~Hz}$ & $40 \mathrm{~Hz}$ \\
\hline
\end{tabular}

All tests have been performed in MATLAB 2017a. VSI frequency is dictated by the user, and the PLL tracks this angle of the grid for the AFE. The system will ramp up in frequency starting at $300 \mathrm{~Hz}\left(f_{n_{\min }}\right)$ all the way to $800 \mathrm{~Hz}\left(f_{n_{\max }}\right)$ where a step $1 \mathrm{~kW}$ load is initiated during the ramp at $0.07 \mathrm{~s}$. To ensure
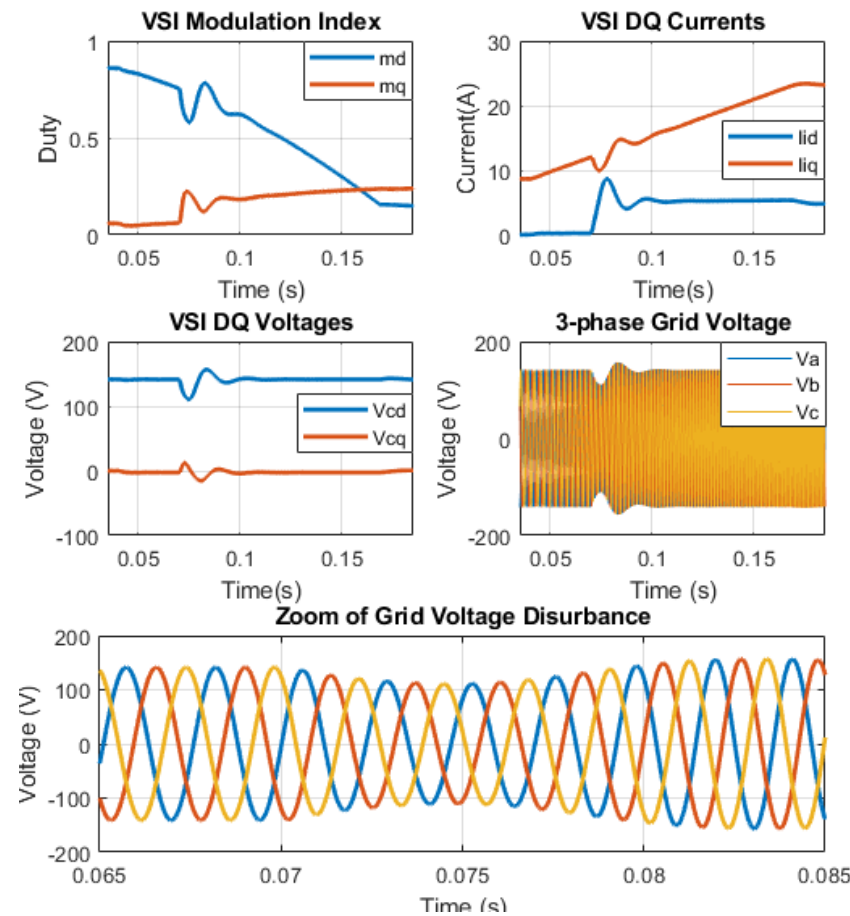

(a) VSI under PI control during frequency transient
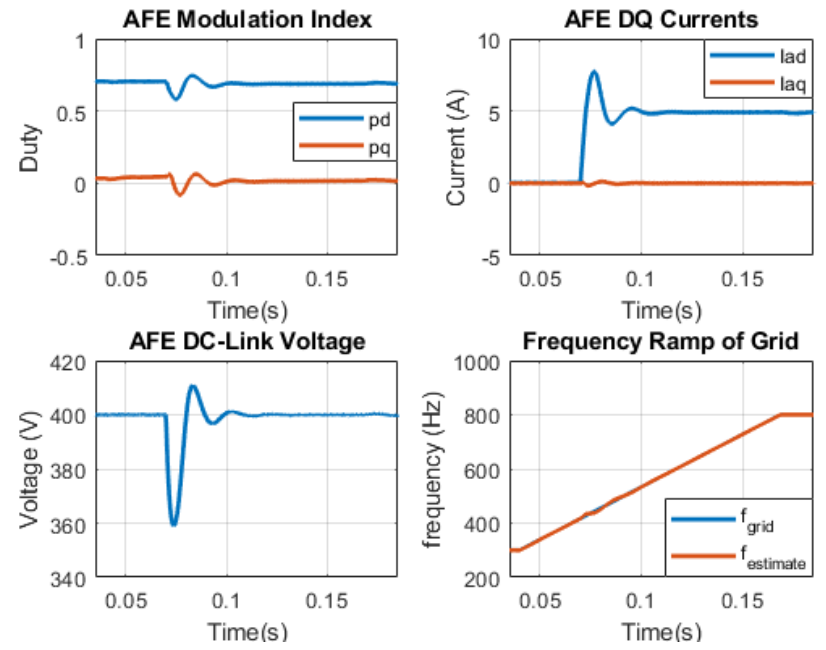

(b) AFE under PI control during frequency transient

Fig. 4: Simulation Results. (d-axis terms noted in Blue, and $\mathrm{q}$ in red for dq terms)

good tracking for the PLL of the grid angle during the ramp, the PLL bandwidth $\omega_{p l l}$ is set to $300 \mathrm{~Hz}$ in both tests.

Upon analyzing Figure 4, the PI control shows little in the way of performance degradation during the frequency transient as expected due to the control not being frequency dependant. However, at the step $1 \mathrm{~kW}$ load disturbance, distortions and interactions occur across all states of the system. Most notably the VSI output voltage $V_{c d}$. A distortion of $30 \mathrm{~V}$ occurs on this state which in turn results in large transients on the grid. In applications such as aircraft grids, this is highly undesirable where a constant grid voltage is to be expected for all types of disturbances. This is largely due to interactions with the AFE converter, whose DC-Link suffers a $40 \mathrm{~V}$ undershoot 

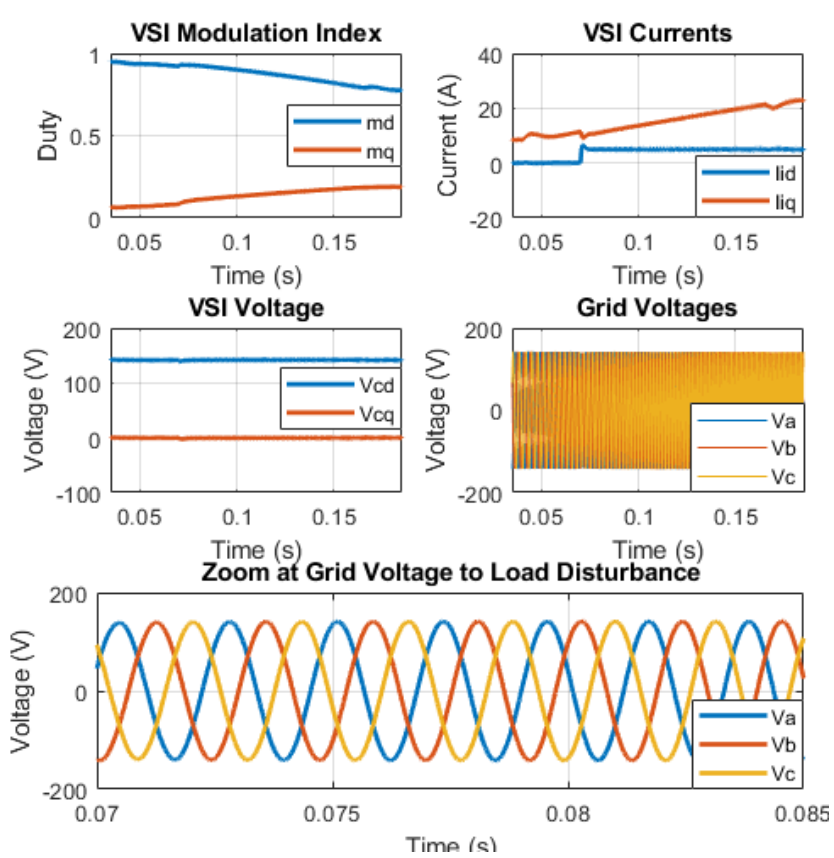

(a) VSI Behaviour during frequency transient
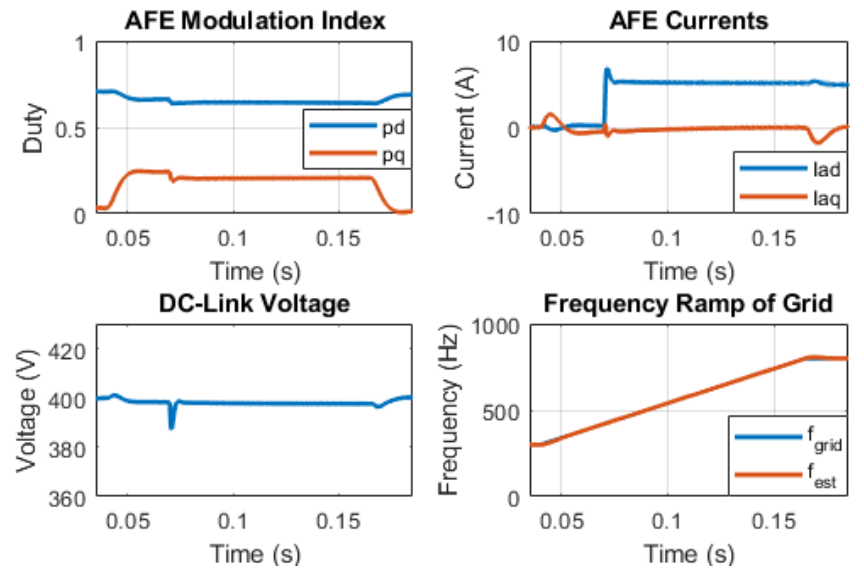

(b) AFE Behaviour during frequency transient

Fig. 5: Simulation Results. (d-axis terms noted in Blue, and $\mathrm{q}$ in red for $\mathrm{dq}$ terms)

during load disturbance, which in turn influences the large overshoots on the AFE current $I_{a d}$. All states reach stable equilibrium within $30 \mathrm{~ms}$. Figure 5 shows the results achieved from the optimal $\mathrm{H}_{2}$ polynomial gain controller, where the gains were generated using the HIFOO toolbox [11]. What can be immediately seen is that across the AFE states, faster and smaller transients are observed. The $H_{2}$ control incurs only a $10 \mathrm{~V}$ undershoot for the DC-Link voltage, and reaches equilibrium again within $5 \mathrm{~ms}$, which is $6 \mathrm{x}$ faster than that of the PI control. However, most importantly are the states of the VSI. It can be observed that minimalistic interactions have occurred. By incorporating dynamic interactions between converters into the controller design, the VSI has had little effect imposed on it due to the AFE state disturbances. Grid voltages have remained largely constant during the frequency and load transients, with the remaining VSI states remaining largely unaffected. The main drawback to the $H_{2}$ control in this application is due to the fact the controller is frequency dependent, and small errors of around $1 \mathrm{~V}$ on the DC-Link, and $0.2 \mathrm{~A}$ on both $I_{a d}$ and $I_{a q}$ occur during the frequency transient. However, once the transient is over, all terms reach equilibrium with zero-steady state error, showing stable and fast performance across the full frequency range.

\section{Conclusion}

This paper has presented a novel approach to optimal decentralized controller design for a simplified embedded grid system based on MEA applications. The new approach was compared to a traditional method of design and the results compared. The proposed approach showed fast performance and little influence due to the frequency transient except for some small but relatively negligible errors which incur due to the frequency dependence of the proposed control. During a frequency transient, a step load disturbance was applied which showed the strengths of an optimal decentralized control, where disturbances on one converter, did not influence or degrade the performance of other subsystems, presenting cross-coupling mitigation. The control enabling self-tuning to system frequency changes off-line makes the controller computationally lightweight, easy to implement, and dynamic performance superior to that of traditional controls schemes.

\section{REFERENCES}

[1] X. Roboam, B. Sareni, and A. De Andrade, "More electricity in the air: Toward optimized electrical networks embedded in more-electrical aircraft," IEEE industrial electronics magazine, vol. 6, no. 4, pp. 6-17, 2012.

[2] D. Dewar, A. Formentini, and P. Zanchetta, "Automated and scalable optimal control of three-phase embedded power grids including pll," in Energy Conversion Congress and Exposition (ECCE), 2017 IEEE. IEEE, 2017, pp. 4252-4259.

[3] W. Rohouma, P. Zanchetta, P. W. Wheeler, and L. Empringham, "A fourleg matrix converter ground power unit with repetitive voltage control," IEEE Transactions on Industrial Electronics, vol. 62, no. 4, pp. 2032 2040, 2015.

[4] D. Dewar, K. Li, A. Formentini, P. Zanchetta, and P. Wheeler, "Performance analysis of h2 optimally controlled three-phase grids," in 2018 IEEE Energy Conversion Congress and Exposition (ECCE). IEEE, 2018, pp. 2258-2264.

[5] M. Cucuzzella, G. P. Incremona, and A. Ferrara, "Decentralized sliding mode control of islanded ac microgrids with arbitrary topology," IEEE Transactions on Industrial Electronics, vol. 64, no. 8, pp. 6706-6713, 2017.

[6] B. Majmunović, T. Dragičević, and F. Blaabjerg, "Multi objective modulated model predictive control of stand-alone voltage source converters," IEEE Journal of Emerging and Selected Topics in Power Electronics, 2019.

[7] A. Formentini, D. Dewar, P. Zanchetta, P. Wheeler, D. Boroyevich, and J.-L. Schanen, "Optimal control of three-phase embedded power grids," in Control and Modeling for Power Electronics (COMPEL), 2016 IEEE 17th Workshop on. IEEE, 2016, pp. 1-6.

[8] J. Doyal, K. Glover, P. Khargoneker, and B. Francis, "State space solutions to standard $\mathrm{h} 2$ and $\mathrm{h}^{\sim}$ control problems [j]," IEEE Trans Automat Control, vol. 34, no. 8, pp. 831-847, 1989.

[9] L. Zhongwen, Z. Chuanzhi, Z. Peng, Y. Haibin, L. Shuhui, and B. Jing, "doyal1989state," IEEE Trans Industiral Electronics, vol. 64, no. 5, pp. 3862-3872, 2017.

[10] J. Pérez, S. Cóbreces Álvarez, R. Griñó Cubero, and F. J. Rodriguez Sanchez, "Hinf current controller for input admittance shaping of vsc-based grid applications," IEEE transactions on power electronics, vol. 32, no. 4, pp. 3180-3191, 2017.

[11] D. Arzelier, D. Georgia, S. Gumussoy, and D. Henrion, "H2 for hifoo," arXiv preprint arXiv:1010.1442, 2010. 